\title{
Evaluación de resultados de la atención médica ambulatoria de niños y adolescentes
}

\author{
Ernesto Medina L. ${ }^{\text {; }}$, Ana M. Kaempffer R. ${ }^{1}$; Edith Cornejo A. ${ }^{2}$; Enrique Hernández A. ${ }^{3}$
}

\begin{abstract}
Resumen
Objefive: conocer los resultados olejados de la atención médica ambulabria explorando la situación de pacientes consulionles de alención primarió nio urgente procedentes de consultorios del sector público del óreo norte de Sontiago, 30 dias después de haber sido atendidos. Pacientes y métodos: 412 niños y adolescentes consuliantes fueron visitados en sus doricilios, idensfificando su situación; sonos y recuperados; camplicados o con secuelas; hospitalizoción; follecidos. Las visiras fueron realizadas por internos de medicina adiestrados para el propósito. Resultodos: los pacientes habian consuliado mayorikariamente por problemas respiratorios e infecciosas trasmisibles con un lapso mecio de 3 dias entre aparición de sintomas y consulta. Se emplearon los sistemas de apoyo diagnóstico y terapéutiço habituales. Los niños pertenecion a grupos socioeconómicos bajos de acuerdo al ingreso farmiliar y escolaridad de las madres. Treinta dias después de la consulta, el $80.1 \%$ se habian recuperado toralmenle, $17,7 \%$ renia complicaciones o secuelas y $2,4 \%$ requirieron hospitalización. Ninguno había fallecido. Los resullados se asocian significativamente al tipo de palo:ogía otendida, a la duración de la enfermedad, el cumplimienlo de las indiccciones posiconsulta, la calidad de la aler.ción y el nivel de vida de los pocientes. No se encontró rejación con la edad, género, precocidad de la primera consulta o número total de consulias. Conclusiones: la capacidad resolutiva real de la alencién primaria alcanza a un $60 \%$ y varia signilicalivamente de ocuerdo al diagnóstico, al cumplimierio de indicaciones y el nivel de vida de los pacienles.
\end{abstract}

(Palabras clave: alerición cmbulatoria, primaria, nin̄os, adolescentes.|

\section{Primary care evaluation: a follow-up of children and adolescents visits}

Objetive: 10 assess health condition thity days afler medical visit of children from nonurgent primary care centers of Santiago. Awethad: 412 chiltren and adelescenls were visited at their homes evalualing their health condition identifying recovery from illress: complications or sequelae; hospilalization; death. Results: respiratory titnesses and communicoble diseases were the most frequent cause of medical visii. Three days was the average lime between symptems and consultotion. Polienls received usual diognostic and therapeutic procedures. Children's families were of low standard of living. Thisty days after medical visit an $80.1 \%$ out of tolol cases were recovered, $17.7 \%$ had complications or sequeloe and $2.4 \%$ required hosptatization. No deaths were registered. Results are significantly asscariared to type of pathology, tength of illress, medical indicalion accomplishment and children's standord of living. Conclusions: good results of primary care cre observed in $30 \%$ of patients varying significantly according to diagnosis, accomplishrnent of medical indications and standard of living of the palienls.

(Key words. ambulatory cere, children, adolescents.)

1. Escuela de Salud Pública, Facultad de Medicina, Universidad de Chile.

2. Enfermera Universitaria. Escuela de Salud Pública, Facultad de Medicina. Universidad de Chile.

3. Profesor de Estadística. Escuela de Salud Pública, Facultad de Medicina, Universidad de Chile.

Este trabajo forma parte de la ìnvestigación "Atención médica: evaluacion de resultados en el corto y mediano plazo" financiada por el Proyecto FONDECYT No 1970249 1997.
La atención médica que se da a las comunidades constituye un hecho complejo que se suele evaluar en términos cuantitativos (número absoluto de atenciones; consultas u hospitalizaciones por persona, etc.), cualitativos (calidad de la atención); o de resultados inmediatos (proporciones de pacientes dados de alta vivos). Menos frecuentemente se realizan evaluaciones de resultados a plazos mayores, salvo cuando el 
problema así lo amerita, como ocurre en el caso de los tumores malignos.

En la literatura médica chilena raramente se encuentran evaluaciones de la atención médica, considerando lo que ocurre después del alta de Jos pacientes hospitalarios o de la consulta ambulatoria. Por esta razón nos interesó desarrollar un proceso de investigación que evaluara los resultados alejados de la atención médica considerando la condición alcanzada por los pacientes en períodos alejados del momento de atención.

\section{Pacientes y métodos}

La investigación ha considerado el estudio de una muestra de J 000 enfermos ingresados en 1997 a los hospitales San José, Roberto del Río y Clínico de la Universidad de Chile, que fueron visitados en sus domicilios 30, 180 y 365 dias después del alta. Se exploró paralelamente una muestra aleatoria de 1000 pacientes ambulatorios, consultantes de atención primaria no urgente. procedentes de consultorios del sector püblico del área norte de Santiago. Los pacientes fueron visitados en sus domicilios, examinando la situación existente treinta días después de haber sido atendidos, identificando si se encontraban sanos y recuperados; presentaban complicaciones o secuelas: habian sido hospitalizados, o habían fallecido. Los casos provienen homogeneamente de los diversos meses del año. Las visitas domiciliarias fueron realizadas por internos de la carrera de Medicina, especialmente adiestrados para el propósito.

De los 1000 pacientes ambulatorios un total de 412 fueron niños y adolescentes menores de 20 antos, cuyo análisis constituye el motivo de esta comuniçación. Los enfermos corresponden a 67 lactantes menores de un año: 129 niños de 1 a 4 anes: 112 de 5 a 9 años y 104 adolescentes de 10 a 19 años. Para el análisis de las patologíos se utilizo la Clasificación Internacional de Enfermedades I. El análisis estadístico emplé preferentemente la prueba de $\mathrm{Ji}^{2}$, considerando como significativos valores inferiones a $p$ $<0,05$.

\section{Resultados}

La mayoría de la pacientes presentaban diversas expresiones de problemas del aparato respiratorio $(66 \%)$, o sufrían alguna enfermedad infecciosa trasmisible (12\%). Otros trastornos, en frecuencias decrecientes, fueron afecciones del aparato locomotor, de los órganos de los sentidos, traumatismos, enfermedades de la piel, mentales, digestivas y otras.

Las visitas domiciliarias permitieron cuantificar la duración de las enfermedades atendidas. Se trató de procesos de alguna duración, con un promedio de 16,6 días y mediana de 10 días. El tiempo entre la iniciación de los síntomas y la primera consulta tuvo una mediana de tres días.

La mitad de los pacientes seguidos durante 30 días ( $51 \%$ ) hizo una sola consulta; en $38,3 \%$ se agregó una segunda y $10,7 \%$ oscilaron entre 3 y 7 consultas. El promedio global fue de 1,74.

Respecto a manejo de los pacientes, $17,5 \%$ requirieron apoyo externo, constatándose necesidad de exámenes de laboratorio en 8,3\% de ellos, agregándose interconsulta $(5,3 \%)$, radiología $(2,9 \%)$, psicoterapia $(0.5 \%)$ y hospitalización $(0,5)$.

Los medicamentos empleados preferentemente fueron antibióticos (27,5\% de los casos), antipiréticos $(18,0 \%)$, broncodilatadores y descongestionantes $(14,2 \%)$, antialérgicos $(8,5 \%)$ $y$ antiinflamatorios $(7,4 \%)$. La vitamina $\mathrm{C}$ fue empleada en $10 \%$ de los casos. La mitad de los pacientes recibio dos o más fármacos, la otra, monoterapia. En la mayoría de los casos se siguieron totalmente $(86,5 \%)$ las indicaciones dadas al paciente, observándose $9,5 \%$ de cumplimiento parcial y $4,0 \%$ de no cumplimiento.

Los niños pertenecen a grupos de bajo nivel de vida. El ingreso familiar mensual fue inferior a $\$ 200.000$ en $95,1 \%$ de los casos y a $\$ 100.000$ en $66 \%$. Casi un cuarto $(23,3 \%)$ de las madres tenían sólo entre 0 y 4 afos de instrucción, $30,6 \%$ tenían 5 a 8 años; $35,4 \%$ ensenanza media y $9,2 \%$ uno o más años de educación universitaria; en $1,5 \%$ no se dispuso de información.

La visitas domiciliarias revelaron que un mes después de la atención $80,1 \%$ de los niños se habian recuperado totalmente del problema que motivó la consulta, $17,5 \%$ presentaban complicaciones o secuelas y $2,4 \%$ requirieron hospitalización (tabla 1). Ninguno de los casos había fallecido. Se registraron mayores porcentajes de recuperación completa en los niños menores de 5 años $(83,7 \%)$ que en los mayores de dicha edad $(76,6 \%)$, aun cuando la diferencia no alcanza significado estadístico. No hubo tampoco diferencias según sexo.

Como se aprecia en la tabla 2, la proporción de recuperación completa a 30 días varía según el tipo de patología atendida. Difieren significativamente ( $p<0,01$ ) los pacientes consultantes por enfermedades infecciosas o por problemas de órganos de los sentidos de aquellos cuya patología fue de tipo mental o del aparato locomotor. 


\section{Tabla 1}

Resultados a 30 días según edad de los niños

\begin{tabular}{rccccc}
\hline Edad (años) & n casos & \multicolumn{2}{c}{ Resul a d os } \\
Sanos & $\begin{array}{c}\text { Complicaciones } \\
\text { o secuelas }\end{array}$ & Hospitalización & Total \\
\hline Total & 412 & 80,1 & 17,5 & 2,4 & 100 \\
1 & 67 & 84,8 & 9,1 & 6,1 & 100 \\
$1-4$ & 129 & 83,1 & 16,9 & - & 100 \\
5.9 & 112 & 76,7 & 17,9 & 5,4 & 100 \\
$10-19$ & 104 & 76,5 & 23,5 & - & 100 \\
\hline
\end{tabular}

Tabla 2

Resultados a 30 días según tipo de patología

\begin{tabular}{|c|c|c|c|c|c|}
\hline TIpo de patología & n casos & $\begin{array}{c}\text { Samos } \\
\text { recuperados }\end{array}$ & $\begin{array}{c}\text { Resu I tad os } \\
\text { Complleaciones } \\
\text { o secuelas }\end{array}$ & Hospitalización & Total \\
\hline Total & 412 & 80.1 & 17.5 & 2,4 & 100 \\
\hline Infecciosas trasmisibles & 48 & 91,6 & 4,2 & 4,2 & 100 \\
\hline Mentales & 12 & 50,0 & 50,0 & - & 100 \\
\hline Organos de los sentidos & 14 & 100,0 & - & - & 100 \\
\hline Respiratorias & 272 & 82,4 & 14,7 & 2,9 & 100 \\
\hline Digestiyas & 10 & 80,0 & 20,0 & - & 100 \\
\hline Piel & 13 & 83,3 & 16,7 & - & 100 \\
\hline Aparato locomotor & 18 & 33,3 & 66,7 & - & 100 \\
\hline Traumatismos & 14 & 71,4 & 28,6 & - & 100 \\
\hline Otras & ॥ & 72,7 & 17,3 & - & 100 \\
\hline
\end{tabular}

La duración de la enfermedad fue significativamente menor en los pacientes recuperados (promedio 13,4 días) que en los complicados $(30,0)$ u hospitalizados (42,0 días).

La precocidad en la consulta no determinó mejores resultados a 30 días. Cuando ella se realiza en los primeros 4 días de evolución los resultados favorables son superiores a los observados en plazos mayores, pero la diferencia no alcanza significado estadístico.

El número total de consultas por problema no se asocia con los resultados alejados. El promedio global de consultas fue de 1,74 , observándose valores de 1,67 en los pacientes recuperados; 1,77 en los complicados o con secuelas y 4,2 en aquellos que requirieron hospitalizacion. Las diferencias no son significativas.

El cumplimiento de las indicaciones formuladas en la consulta constituye un factor asociado a los resultados observados 30 días después (tabla 3). En este análisis no se incluyen 10 casos de hospitalización por cuanto fueron ingresados como consecuencia inmediata de la consulta. Cuando el cumplimiento de las indicaciones es completo, los resultados son significativamente mejores $(p=0,0 \mathrm{l})$.

En los niños de familias de bajo ingreso (menos de $\$ 100.000$ mensuales) asociado a baja escolaridad materna ( 0 a 4 años de escolaridad) se obtienen peores resultados que en aquellos que no pertenecen a este grupo (tabla 4). Los niños de nivel socioeconómico más bajo tjenen una proporción significativamente superior de enfermos no recuperados y de hospitalizados ( $p<0,059$. En contraste, en los enfermos pertenecientes al sistema previsional de FONASA los resultados no difieren con los de otros sistemas previsionales. 
Tabla 3

Resultados a 30 días según cumplimiento de indicaciones

\begin{tabular}{lccccc}
\hline Cumplimiento & n casos & $\begin{array}{c}\text { Sanos } \\
\%\end{array}$ & $\begin{array}{c}\text { Resultados } \\
\text { Complicaciones } \\
\text { o secuelas } \%\end{array}$ & $\begin{array}{c}\text { Hospitalización } \\
\%\end{array}$ & $\begin{array}{c}\text { Total } \\
\%\end{array}$ \\
\hline $\begin{array}{l}\text { Total } \\
\text { Parcial o no cumplimiento }\end{array}$ & 348 & 85.1 & 14,9 & - & 100 \\
\hline Total & 54 & 57,9 & 42,1 & - & 100 \\
\hline
\end{tabular}

\section{Tabla 4}

Resultados según nivel socioeconómico familiar

\begin{tabular}{|c|c|c|c|c|c|}
\hline $\begin{array}{l}\text { Condlción } \\
\text { socioeconómica }\end{array}$ & n casos & $\begin{array}{c}\text { Sanos } \\
\%\end{array}$ & $\begin{array}{c}\text { Result a đos } \\
\text { Complicaciones } \\
\text { o secuelas \% }\end{array}$ & $\underset{\%}{\text { Hospitalización }}$ & $\begin{array}{c}\text { Total } \\
\%\end{array}$ \\
\hline $\begin{array}{l}\text { Bajo nivel" } \\
\text { Oiros casos" }\end{array}$ & $\begin{array}{r}70 \\
336\end{array}$ & $\begin{array}{l}68,2 \\
83,0\end{array}$ & $\begin{array}{l}25,0 \\
{[5,]}\end{array}$ & $\begin{array}{l}6,8 \\
1,3\end{array}$ & $\begin{array}{r}100 \\
100 p-0,02\end{array}$ \\
\hline Total"** & 406 & 80,1 & 17,5 & 2,4 & 100 \\
\hline
\end{tabular}

* Escolaridad materna entre 0-4 años de instrucción e ingreso economico mensual inferior a \$100.000

** Orras condiciones

*** Hubo 6 casos con información incompleta

En la visita domiciliaria practicada 30 días después de la primera consulta se consultó la opinión respecto a calidad de la atención recibida por el niño (tabla 5). En $68 \%$ de los casos fue buena o muy buena, registrándose $22 \%$ "regulares". La evolución a 30 días observada en los casos de calificación deficiente fue significativamente diferente a la registrada en los otros tres grupos $(\mathrm{p}<0,01)$.

\section{Comentario}

Empleando como método el conocimiento de resultados alejados de los enfermos atendidos, la capacidad resolutiva de la atencion ambulatoria se ubica aproximadamente en $80 \%$ de la demanda satisfecha de atención. La cifra es algo inferior a la obtenida con la estimación propues- ta por Symeant ${ }^{2}$ y seguida por otros ${ }^{3}$, que supone resueltos los casos que no requirieron referencia a otro nivel de atención, para exámenes diagnósticos o interconsulta.

Esta muestra tiene atributos similares a las estudiadas en Santiago sobre morbilidad y atención médica de la población ${ }^{4-6}$ en lo relativo a frecuencias relativas de diversos tipos de afecciones o estructura socioeconomica de las familias.

Hemos encontrado que los resultados de la atención no están asociados a la edad, género, precocidad de la primera consulta, o al número total de consultas por episodio de enfermedad. A la inversa, se asocian significativamente al tipo de enfermedad atendida, a la duración total de ella, el cumplimiento de las indicaciones postconsulta, la calidad de la atención y el nivel de vida de los pacientes. 
Tabla 5

Resultados según opinion de las madres sobre calidad de la atención

\begin{tabular}{|c|c|c|c|c|c|c|}
\hline \multirow{2}{*}{$\begin{array}{l}\text { Opinión sabre } \\
\text { calidad }\end{array}$} & \multirow[b]{2}{*}{$\mathbf{n}$} & \multirow{2}{*}{$\begin{array}{c}\text { n casos } \\
\text { क del total }\end{array}$} & \multirow[b]{2}{*}{$\begin{array}{c}\text { Sanos } \\
\%\end{array}$} & \multicolumn{2}{|c|}{ Resultados } & \multirow[b]{2}{*}{$\underset{\%}{T}$} \\
\hline & & & & $\begin{array}{l}\text { Complic. o } \\
\text { secuelas \% }\end{array}$ & $\begin{array}{l}\text { Hospita- } \\
\text { lizacion\% }\end{array}$ & \\
\hline Muy buena & 78 & 18,9 & 80,0 & 15,0 & 5,0 & 100 \\
\hline Buena & 202 & 49.0 & 82,0 & 16,0 & 2,0 & 100 \\
\hline Regular & 92 & 22,3 & 80.4 & 17,4 & 2,2 & 100 \\
\hline Deficiente & 28 & 6,8 & 50,0 & 42,9 & 7,11 & 100 \\
\hline Sin opinion & 12 & 2,9 & - & - & - & 100 \\
\hline Total & 412 & 100.0 & 80.1 & 17.5 & 2,4 & 100 \\
\hline
\end{tabular}

p azar $<0,01$

Resulta en cierto modo paradójico que la magnitud de resultados favorables no esté vinculada a la precocidad de la primera consulta y al número total de consultas producidas por el episodio de enfermedad. En cambio era esperable encontrar significativas diferencias de resultado según el tipo de trastomo o el cumplimiento de las indicaciones formuladas por el médico para el período postconsulta.

La relación inversa entre salud materno-infantil y nivel de vida es conocida desde antiguo. Ella está avalada por las experiencias relativas a la mortalidad del lactante y en menor grado del preescolar ${ }^{7}$. La frecuencia y tipo de enfermedad de una población, según los hallazgos nuestros en Santiago ${ }^{8.9}$, muestran un significativo exceso de enfermedad aguda (2,5 veces) y de traumatismos ( 4 veces) en los grupos de pobreza extre$\mathrm{ma}$, esto es con ingresos económicos que no alcanzan para resolver las necesidades nutricionales básicas. Este estudjo agrega a las evidencias anteriores los peores resultados a un mes plazo en el grupo de menor nivel tanto económico como educacional.

\section{Referencias}

1. Organización Panamericana de la Salud. Clasificación estadistica internacional de enfermedades y problemas relacionados con la salud. Décima revisión. CIE-10. Public Cientif OPS N5554, 1995. Washington DC., EE.UU.

2. Symeant S: Capacidad resolutiva de la demanda de atención de monbilidad a nivel primario. Boletín OPS 1984; 97: I25-14I

3. Medina E: Atención primaria del adulto en Chile. Rey Med Chile 1992; 120: 505-513.

4. Kaempfer AM, Medino $E$, Cornejo $E$, Hernández $E$, Wall $V$ : Morbilided infantil y del adolescente en Santiago 1993-1994. Rev Chil Pediatr 1995: 66: 50-54.

5. Kaempffer AM. Medinu E, Comejo E. Hernández E: Morbilidad y atención médica del adolescente. Cuadernos Médico Sociales 1996: 37: 60-65.

6. Medina E, Kaempffer AM. Comejo E, Hernúndez E. Wall V: Atención de salud de nif́os y adolescentes de Santiago 1993-1994. Rev Chil Pediatr 1995; 66: 44-49.

7. Kaenyffer $A M$, Medina $E$ : La salud infantil en Chile durante la década del setenta. Rev Chil Pediatr 1982; 53: 468-472.

8. Medina E, Kaempffer AM. Cunsille JF, Medina R: Atención de salud en el Gran Santiago según niveles de ingreso económico. Cuadernos Médico Sociales 1985; 26: 95-102.

9. Medina E. Kaentpfer AM, Cornejo E, Hernández E, Wall V: Caracteristicas y manejo de la morbilidad en Santiago 1993. Rev Med Chile 1994; 122: 1421-1427. 